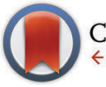

CrossMark \&lickfor updates

Cite this: Chem. Commun., 2016, 52,10747

Received 13th July 2016, Accepted 5th August 2016

DOI: $10.1039 / c 6 c c 05747 \mathrm{k}$

www.rsc.org/chemcomm

\section{Total synthesis of dehaloperophoramidine using a highly diastereoselective Hosomi-Sakurai reaction $\dagger$}

\author{
Ross. P. Wilkie, $\ddagger^{a}$ Andrew R. Neal,$\ddagger^{a}$ Craig A. Johnston, ${ }^{a}$ Nicholas Voute, ${ }^{a}$ \\ Christopher S. Lancefield, ${ }^{a}$ Matthew D. Stell, ${ }^{a}$ Federico Medda, ${ }^{a}$ Edward F. Makiyi, ${ }^{a}$ \\ Emma M. Turner, ${ }^{a}$ O. Stephen Ojo, ${ }^{a}$ Alexandra M. Z. Slawin, ${ }^{a}$ Tomas Lebl, ${ }^{a}$ \\ Peter Mullen, ${ }^{b}$ David J. Harrison, ${ }^{b}$ Chris M. Ireland ${ }^{c}$ and Nicholas J. Westwood ${ }^{\star a}$
}

\begin{abstract}
The synthesis of dehaloperophoramidine, a non-halogenated derivative of the marine natural product perophoramidine, and its biological activity towards HCT116, HT29 and LoVo colorectal carcinoma cells is reported. $A[3,3]$-Claisen rearrangement and an epoxide opening/ allylsilylation reaction installed the contiguous all-carbon quaternary stereocentres with the required relative stereochemistry.
\end{abstract}

The natural product (+)-perophoramidine (1) was isolated by Ireland from the marine ascidian Perophora namei. ${ }^{1}$ The authors also reported the structure of dehaloperophoramidine (2), which they obtained via transfer hydrogenation of (+)-1. These interesting alkaloids are structurally related to a more complex family of natural products that includes communesin F (3). ${ }^{2}$ Targets, including 1, 2 and $\mathbf{3}$, that possess contiguous all-carbon quaternary stereocentres present a significant synthetic challenge. ${ }^{3}$ The presence of this structural unit and the anticancer activity reported for $\mathbf{1}$ has led to several total and formal syntheses. ${ }^{4}$ Previous syntheses of 2 have involved a spirocyclisation of a 2-thiotryptamine analogue, ${ }^{5}$ a dearomatising arylation of a quinoline ${ }^{6}$ and an efficient synthesis from a commercially available indigo dye. ${ }^{7}$

In work aimed at the synthesis of the communesins, we have explored a [3,3]-Claisen approach to establish the required C7 stereocentre in 3 (Fig. 1). ${ }^{8}$ In the first part of this new report we describe a significant extension of this work resulting in the large scale synthesis (62 grams) and resolution of a novel ketone using our [3,3]-Claisen rearrangement method (Scheme 1). This highly efficient sequence, that does not require chromatographic

\footnotetext{
${ }^{a}$ School of Chemistry and Biomedical Sciences Research Complex, University of St. Andrews and EaStCHEM, St. Andrews, Fife, Scotland, KY16 9ST, UK. E-mail:njw3@st-andrews.ac.uk

${ }^{b}$ School of Medicine, University of St Andrews, St. Andrews, Fife, Scotland, KY16 9TF, UK

${ }^{c}$ Department of Medicinal Chemistry, University of Utah, 30 South 2000 East, Salt Lake City, UT 84112, USA

$\dagger$ Electronic supplementary information (ESI) available. CCDC 1486344 (15), 1478153 (S16), 1478152 (5) and 1478154 (30). For ESI and crystallographic data in CIF or other electronic format see DOI: 10.1039/c6cc05747k

\$ These authors contributed equally.
}
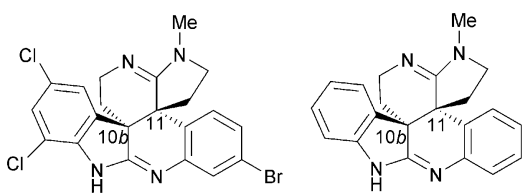

(+)-Perophoramidine (1)

Dehaloperophoramidine (2)

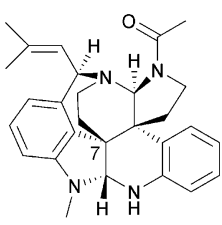

Communesin F (3)

Fig. 1 The structures of $(+)$-perophoramidine (1), (+)-dehaloperophoramidine (2) and communesin $\mathrm{F}(\mathbf{3})$.

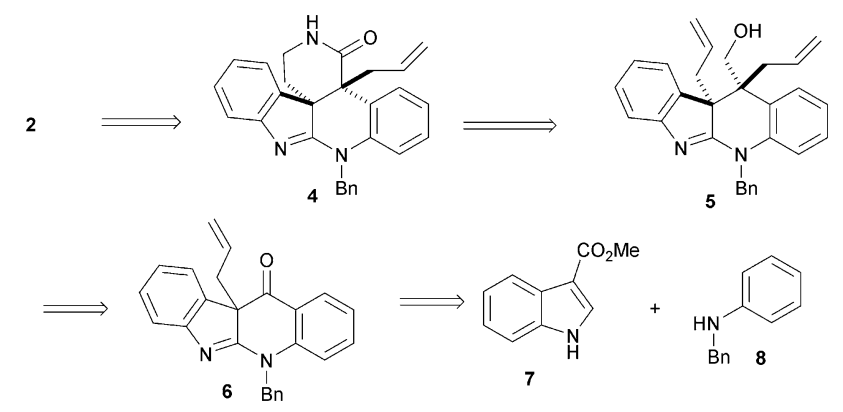

Scheme 1 Retrosynthetic analysis of 2 starting from commercially available $\mathbf{7}$ and $\mathbf{8}$.

purification in any of the first five steps, enables access to enantio-enriched ketones. Whilst we view these ketones as flexible starting points for the synthesis of a number of optically pure complex ring systems, here we report conversion of racemic material to 2 .

Our retrosynthetic analysis of 2 (Scheme 1) identified lactam 4 as the cornerstone of the approach. Lactam 4 was viewed as accessible from diallyl-substituted alcohol 5, which contains both the required $\mathrm{C}-10 \mathrm{~b}$ and $\mathrm{C}-11$ stereocentres. A key challenge in this part of the synthesis was the need to differentiate between the two allyl groups in 5. This was achieved using two different selective iodocyclisation protocols. Whilst this transformation is precedented in simpler systems, ${ }^{9}$ it has been used sparingly in the synthesis of more complex alkaloid-based structures. ${ }^{10}$ 
Alcohol 5 could be prepared from allyl-ketone 6, which itself could be constructed from commercially available 7 and 8 .

The key challenge in the conversion of $\mathbf{6}$ to $\mathbf{5}$ is the incorporation of the second contiguous all carbon stereogenic centre with the required relative stereochemistry. Conversion of the ketone in $\mathbf{6}$ to the corresponding epoxide was planned. Subsequent Lewis acid catalysed epoxide opening followed by trapping with allylsilane was predicted to lead to $\mathbf{5}$ with the approach of the allyl nucleophile occurring from the opposite face to the allyl group that is already present. This reaction, which we refer to as a modified Hosomi-Sakurai reaction, ${ }^{11}$ has been used in natural product synthesis, ${ }^{12}$ but to the best of our knowledge has not been used in the construction of contiguous all-carbon quaternary centres. Alternative approaches to lactam 4 involving the early development of the allyl group in 6 or alkylation of a C11-based ester were explored but were either ultimately unsuccessful or led to significantly longer reaction sequences (data not shown).

The C10b stereocentre in 6 was installed in 5 steps from 7 and $\mathbf{8}$ as described in Scheme 2. After coupling of $\mathbf{7}$ and $\mathbf{8}$ to give $\mathbf{9}$, the tetracyclic system was formed using a high temperature electrophilic aromatic substitution reaction (see Scheme S1 (ESI $\dagger)^{13}$ for additional studies). The cyclisation was scalable up to $100 \mathrm{~g}$ with no detrimental effect on the yield. Functional group manipulations then enabled the preparation, via 10, of the [3,3]-Claisen rearrangement substrate 11. Highly efficient conversion of $\mathbf{1 1}$ to the required novel ketone $\mathbf{6}$ occurred on heating in toluene for 1.5 hours in $88 \%$ yield. This reaction was robust and scalable with $62 \mathrm{~g}$ of 6 being prepared in a single batch and without the need for purification by column chromatography at any stage. Ketone 6 could be resolved by treatment with $(R)$-tert-butanesulfinamide (12) to generate the readily separable diastereomeric imines 13 and $\mathbf{1 4}$ (Scheme 3). This approach was inspired by the reported resolution of a ketone-containing intermediate en route to epiboxidine. ${ }^{14}$ Hydrolysis of imines 13 and 14 with $12 \mathrm{M} \mathrm{HCl}$ in $\mathrm{MeOH}$ gave $(R)-6$ and $(S)-6$ respectively in $>99 \%$ ee after recrystallisation (confirmed by chiral HPLC analysis ${ }^{13}$ ). The absolute configuration of the intermediate imines $\mathbf{1 3}$ and $\mathbf{1 4}$ were assigned following X-ray crystallographic analysis of $\mathbf{1 5}^{\mathbf{1 5}}$ which was obtained following diastereoselective reduction of $\mathbf{1 4}$ with $\mathrm{NaBH}_{4}$ (Scheme 3). The $\mathrm{X}$-ray analysis indicated that the absolute configuration of the $\mathrm{C} 10 \mathrm{~b}$ stereogenic centre in 15 (and hence in 14) was $(R)$.

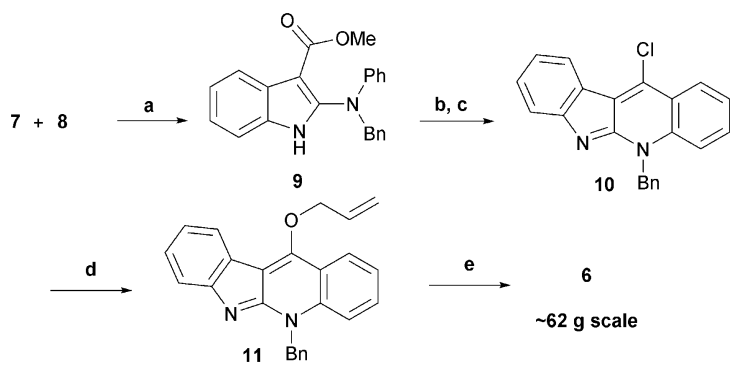

Scheme 2 Construction of key intermediate 6: (a) NCS, DMP, DCM, $0{ }^{\circ} \mathrm{C}$, $2 \mathrm{~h}$; then TCA, $0{ }^{\circ} \mathrm{C}-\mathrm{rt}, 2 \mathrm{~h}, 93 \%$; (b) $\mathrm{Ph}_{2} \mathrm{O}$, reflux, $2.5 \mathrm{~h}, 79 \%$; (c) $\mathrm{POCl}_{3}$, reflux, 95\%; (d) Na (3 eq.), allyl alcohol (5.7 eq.), rt, 18 h, 92\%; (e) PhMe, reflux, $1.5 \mathrm{~h}, 88 \%$.

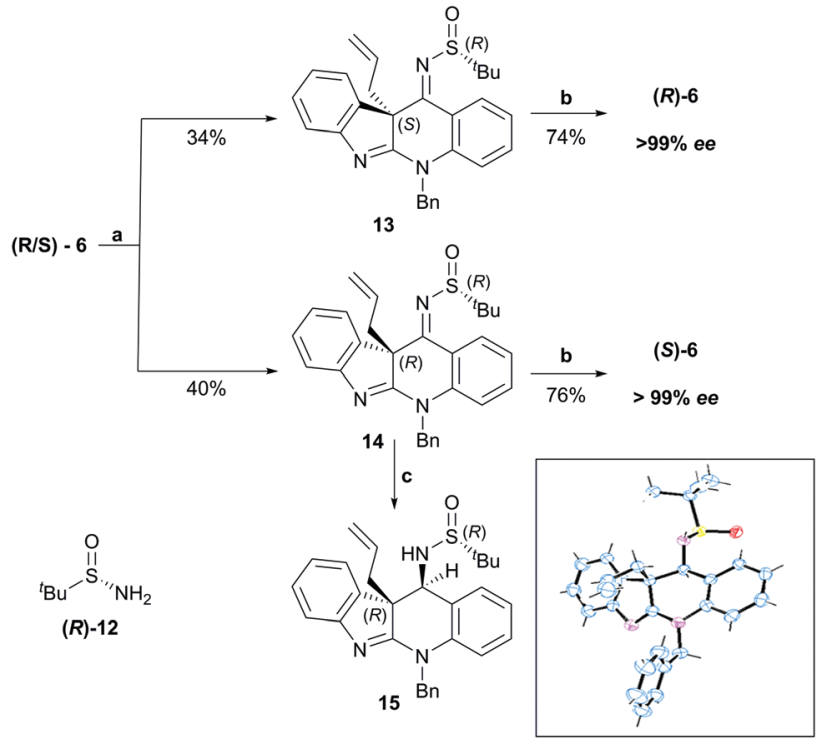

Scheme 3 Obtaining the $(R)$ and $(S)$ enantiomers of ketone 6 from their respective imines 13 and 14 . The ORTEP representation of 15 is also shown and was used to infer the absolute configuration of 14 and (S)- 6 (and hence 13 and (R)-6). Reagents and conditions: (a) $R$-(12) (10 eq.), Ti(OEt) 4 (15 eq.), toluene, $85{ }^{\circ} \mathrm{C}, 9 \mathrm{~h}$; (b) $12 \mathrm{M} \mathrm{HCl}, \mathrm{MeOH}, \mathrm{rt}, 0.5 \mathrm{~h}$; (c) $\mathrm{NaBH}_{4}$ (2.5 eq.), $\mathrm{MeOH}, \mathrm{rt}, 0.5 \mathrm{~h}, 92 \%$.

Hydrolysis of imine 14 therefore provided the $(S)$-enantiomer of 6 (and 13 gave $(R)-6)$. Whilst $(R)-6$ and $(S)-\mathbf{6}$ have been prepared, their conversion to enantio-enriched alternative complex ring systems will be reported in the future. Here we decided to continue the development of a route to 2 using the much larger quantities of $( \pm)-6$ available to us.

With the novel ketone 6 in hand, the ketone functionality was converted to the corresponding epoxide using $\mathrm{ClCH}_{2} \mathrm{I}^{16}$ (see Scheme S2 (ESI $\dagger$ ) for the stereochemical assignment of 16). ${ }^{13}$ Allylsilylation of 16 under Lewis acidic conditions with allylTMS (17) in a modified Hosomi-Sakurai reaction ${ }^{11}$ gave the desired alcohol 5 as a single diastereoisomer (as judged by ${ }^{1} \mathrm{H}$ NMR analysis of the crude reaction mixture). This reaction proved highly robust and scalable with ca. $42 \mathrm{~g}$ of 16 being reproducibly converted to $38 \mathrm{~g}$ of $\mathbf{5}$. X-ray crystallographic analysis of 5 confirmed the required anti-relationship of the two allyl substituents at $\mathrm{C}-10 \mathrm{~b}$ and $\mathrm{C}-11$ (Scheme 4 and Scheme S3, ESI $\dagger$ ). ${ }^{13}$

The challenge of selectively functionalising the two allyl groups in 5 now had to be overcome. A two-step oxidation of $\mathbf{5}$, followed by regioselective iodolactonisation gave $\mathbf{1 8}$ and its epimer at the indicated carbon as an inconsequential mixture of diastereoisomers (Scheme 5, d.r. 3:1). The relative configuration of the newly formed stereocentre in the major isomer 18 was determined by nOe analysis (Scheme S4 and Fig. S2-S4, ESI $\dagger$ ). ${ }^{13}$ Oxidative cleavage on treatment with catalytic $\mathrm{OsO}_{4}$ and $\mathrm{NMO}$ followed by in situ reaction with $\mathrm{PhI}(\mathrm{OAc})_{2}$ gave 19 and its epimer (Scheme 5 and Scheme S5, ESI $\dagger$ ). ${ }^{13}$ After incorporation of the required nitrogen by reductive amination using $( \pm)-\mathbf{1 2}^{17}$ (Scheme 3), retro-iodolactonisation to give the diastereomeric mixture 20, acid-mediated deprotection and subsequent treatment of 21 with HBTU and DIPEA, lactam 4 was obtained. However, cyclisation 

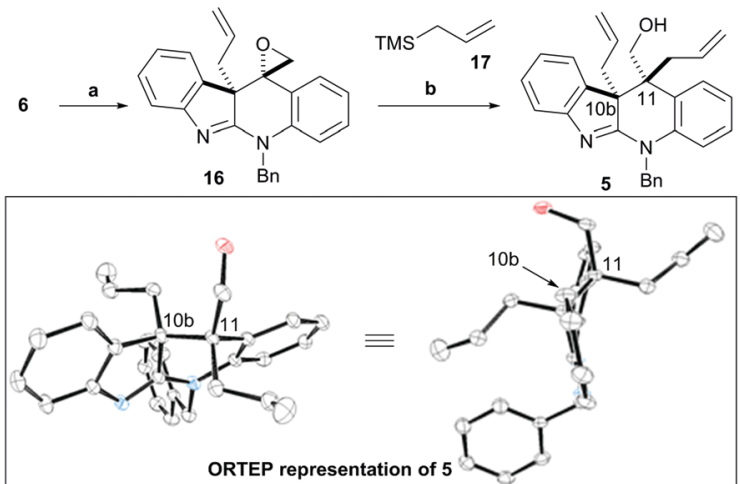

Scheme 4 Construction of all-carbon quaternary stereocentres. The ORTEP representation of $\mathbf{5}$ is also shown. ${ }^{15}$ Reagents and conditions: (a) $\mathrm{ClCH}_{2} \mathrm{I}$ (1.1 eq.), $\mathrm{MeLiBr}\left(2.2 \mathrm{M}\right.$ solution in $\mathrm{Et}_{2} \mathrm{O}, 1.5$ eq.), THF, $-78{ }^{\circ} \mathrm{C}, 2 \mathrm{~h}$; (b) allylTMS (17) (2.5 eq.), $\mathrm{TiCl}_{4}$ (4 eq.), DCM, $-78{ }^{\circ} \mathrm{C}, 78 \%$ (2 steps).
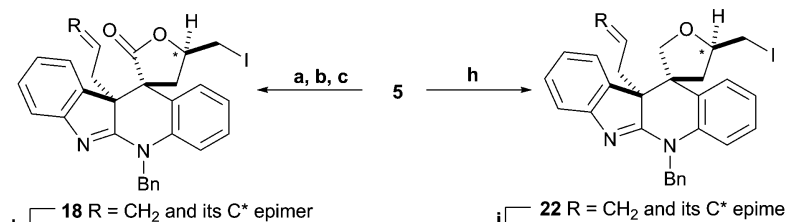

d $\square 18 \mathrm{R}=\mathrm{CH}_{2}$ and its $\mathrm{C}^{*}$ epimer

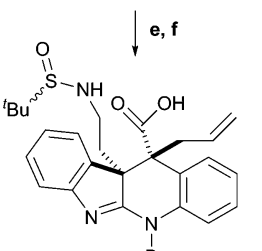

$20^{\mathrm{Bn}}$

g

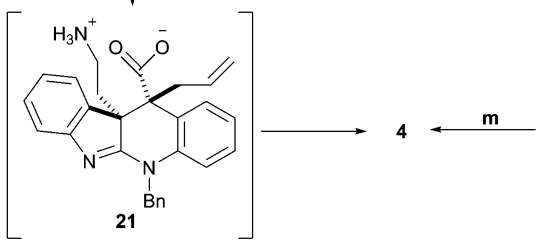

$22 \mathrm{R}=\mathrm{CH}_{2}$ and its $\mathrm{C}^{*}$ epime

$23 \mathrm{R}=\mathrm{O}$ and its $\mathrm{C}^{*}$ epimer
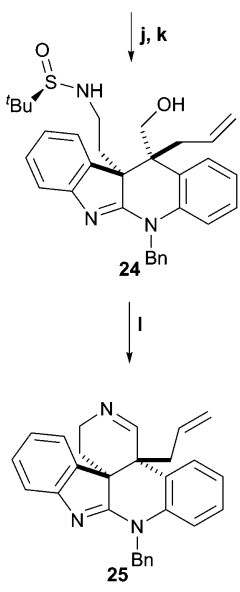

Scheme 5 Two alternative approaches to lactam 4. Reagents and conditions: (a) PCC (1.1 eq.) DCM, rt, 16 h; (b) Jones reagent (1.5 eq.), acetone, rt, 16 h; (c) NIS (1.1 eq.), $\mathrm{NaHCO}_{3}$ (1.1.eq.), DCM, rt, 16 h, d.r. $3: 1,47 \%$, (over 5 steps from 7); (d) $\mathrm{OsO}_{4}\left(0.13\right.$ mol\%), $\mathrm{NMO}$ (1.5 eq.), acetone $/ \mathrm{H}_{2} \mathrm{O}(10: 1), \mathrm{rt}, 18 \mathrm{~h}$, then Phl(OAc) 2 (1.5 eq.), rt, 1.5 h, 86\%; (e) ( \pm )-12 (1.1 eq.), $\mathrm{TiOEt}_{4}$ (3 eq.) $\mathrm{CHCl}_{3}, \mathrm{rt}$, 16 h, then $\mathrm{NaBH}_{4}$ (4 eq. in $\mathrm{MeOH}$ ), 0.5 h, rt, 75\%; (f) $\mathrm{Zn}, \mathrm{EtOH}$, reflux, 16 h, 89\%; (g) $4 \mathrm{M} \mathrm{HCl}$ in dioxane/MeOH $(10: 1)$, rt, $1 \mathrm{~h}$, then HBTU (1.5 eq.) and DIPEA (5 eq.), 16 h, $71 \%$; (h) $\mathrm{I}_{2}$ (2.5 eq.), $\mathrm{K}_{2} \mathrm{CO}_{3}$ (2.5 eq.), rt, $\mathrm{MeCN}, 16$ h, d.r. $11: 1,96 \%$; (i) $\mathrm{OsO}_{4}(0.19 \mathrm{~mol} \%), \mathrm{NMO}$ (1.5 eq.), THF: $\mathrm{H}_{2} \mathrm{O}(9: 1), \mathrm{rt}, 16 \mathrm{~h}$, then $\mathrm{Phl}(\mathrm{OAc})_{2}$ (1.5 eq.), rt, 2 h, 66\%; (j) (R)-16 (1.1 eq.), Ti(OEt) 4 (2 eq.), $\mathrm{CHCl}_{3}, \mathrm{rt}, 16 \mathrm{~h}$, then $\mathrm{NaBH}_{4}$ (5 eq.) in $\mathrm{MeOH}, \mathrm{rt}, 1 \mathrm{~h}, 68 \%$; (k) $\mathrm{Zn}$ (31 eq.), EtOH, reflux, 16 h, 80\%; (l) Dess-Martin periodinane (1.5 eq.), DCM, rt, $0.5 \mathrm{~h}, 52 \%$, (m) $\mathrm{NaClO}_{2}$ (2.5 eq.), $\mathrm{NaH}_{2} \mathrm{PO}_{4}$ (5 eq.), 2-methylbuten-2-ene (10 eq.), THF/ $\mathrm{H}_{2} \mathrm{O}(1.5: 1), \mathrm{rt}, 2$ h, $58 \%$.

of 20 to 4 proved irreproducible (for further discussion see Schemes S6-S8 and Fig. S5, ESI $\dagger){ }^{13}$ An alternative route to 4 was investigated in an attempt to circumvent the reproducibility issue. Di-allyl alcohol 5 underwent a regio- and highly diastereo-selective iodoetherification to give 22 (and its epimer, d.r. 11 :1, Fig. S6, $\mathrm{ESI} \dagger)^{13}$ in the presence of iodine under basic conditions. A one-pot oxidative cleavage of the alkene gave $\mathbf{2 3}$ (and its epimer) which underwent successful reductive amination with $(R)-\mathbf{1 2}$ followed by a retro-iodoetherification in the presence of $\mathrm{Zn}$ to generate the diastereomeric mixture 24. Oxidation of $\mathbf{2 4}$ with the Dess-Martin periodinane $^{18}$ gave 25 in a reasonable yield (52\%) for this relatively complex process which also involves acid-mediated deprotection of the sulfinamide. Interestingly, 25 underwent oxidation with $\mathrm{NaClO}_{2}$ to give the required lactam 4. This transformation was inspired by a report by Tomioka et al. on an unrelated system. ${ }^{19}$ The new approach was robust and overall reduced the length of the reaction sequence to 4 .

Having successfully synthesised the desired lactam 4, its conversion to 2 was completed (Scheme 6). The remaining alkene in lactam 4 was oxidatively cleaved and the resulting aldehyde reductively aminated using $\mathrm{MeNH}_{2} \cdot \mathrm{HCl}$ under basic conditions to give 26 (Scheme 6). Boc protection of $\mathbf{2 6}$ gave $\mathbf{2 7}$ which underwent alkylation with Meerwein's reagent ${ }^{20}$ to give $28 .^{13}$ Deprotection of 28 with trifluoroacetic acid gave 29 which cyclised on refluxing in toluene to give $N$-benzyl-dehaloperophoramidine (30) in excellent yield. X-ray crystallographic analysis of $\mathbf{3 0}$ confirmed the successful formation of the $\mathrm{C}-4^{\prime}$ amidine motif. ${ }^{15}$ The synthesis of 2 was completed by $N$-benzyl deprotection of $\mathbf{3 0}$ via a single electron transfer process with a freshly prepared solution of sodium naphthalenide. ${ }^{21}$

Synthetic 2 was converted to the corresponding TFA salt ${ }^{13}$ and compared to an authentic sample of 2-TFA (Fig. S7-S9, ESI $\dagger$ ). ${ }^{13}$ A doping experiment with authentic 2-TFA and synthetic 2-TFA confirmed that the desired compound had been successfully prepared (see Fig. S8A and B, ESI $\dagger$ for a comparison of a selected region of the ${ }^{1} \mathrm{H}$ NMR analysis carried out in $\left.\mathrm{CD}_{3} \mathrm{OD}\right)$. Superimposition of the ${ }^{1} \mathrm{H}$ NMR of the doped sample with the
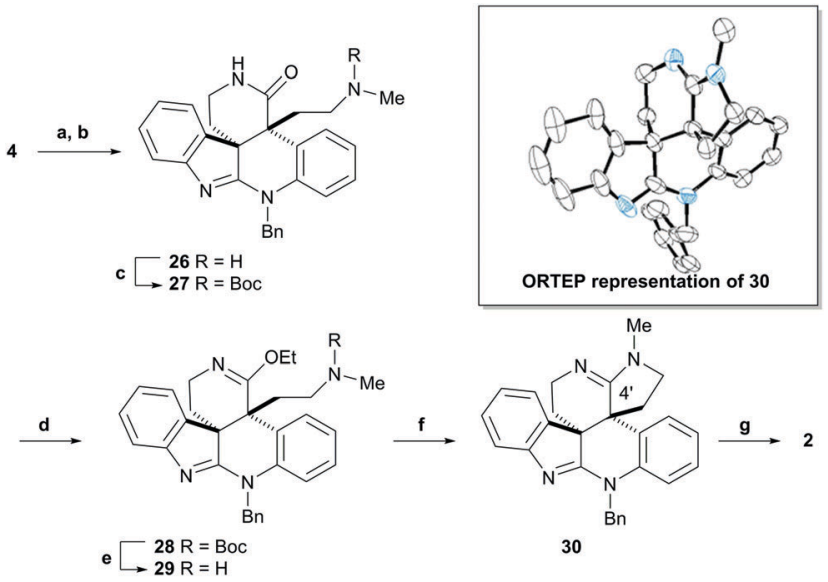

Scheme 6 Completion of the synthesis of $( \pm)-2$. Reagents and conditions: (a) $\mathrm{OsO}_{4}$ (3.2 mol\%), NMO (1.5 eq.), THF/ $\mathrm{H}_{2} \mathrm{O}, \mathrm{rt}, 16 \mathrm{~h}$, then $\mathrm{Phl}(\mathrm{OAc})_{2}$ $1.5 \mathrm{~h}, 62 \%$ (over 2 steps from 25); (b) $\mathrm{MeNH}_{2} \cdot \mathrm{HCl}$ (2.5 eq.), $\mathrm{NaOAc}$ (2.5 eq.), $\mathrm{MeOH}, \mathrm{rt}, 16 \mathrm{~h}$, then $\mathrm{NaBH}_{4}$ (3 eq.), rt, $0.5 \mathrm{~h}$; (c) (Boc) ${ }_{2} \mathrm{O}$ (1.5 eq.), TEA (1.5 eq.), rt, $1 \mathrm{~h}, 44 \%$ (2 steps); (d) Meerwein's reagent ( $1 \mathrm{M}$ solution in DCM, 8 eq.), DIPEA ( 8 eq.), DCM, $0{ }^{\circ} \mathrm{C}-\mathrm{rt}, 2 \mathrm{~h}, 72 \%$; (e) $5 \%$ TFA, DCM, $0{ }^{\circ} \mathrm{C}, 0.5 \mathrm{~h}$; (f) DIPEA (2 eq.), toluene, reflux, $16 \mathrm{~h}, 83 \%$ (over 2 steps); (g) Na/naphthalene (1 $\mathrm{M}$ solution), $\mathrm{THF}, 0{ }^{\circ} \mathrm{C}-\mathrm{rt}, 2 \mathrm{~h}, 50 \%$. 
Effect of 2 on the growth of HCT116, HT29 and LoVo colorectal cancer cells (day 4).

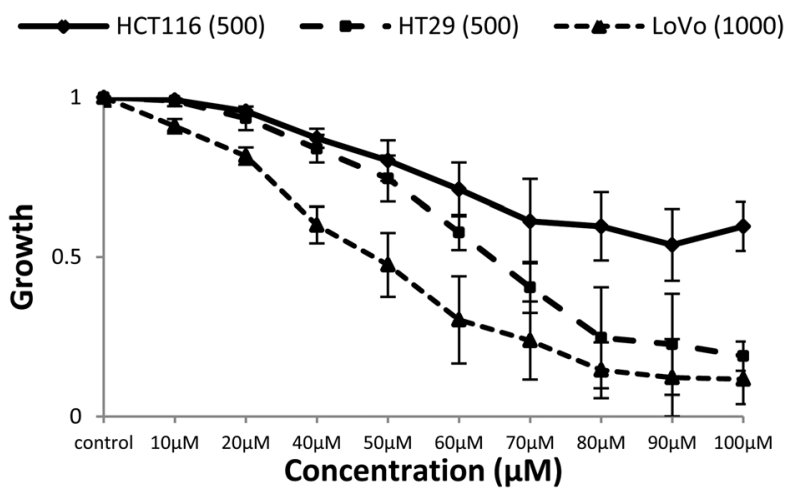

Fig. 2 Dose-response curves for the effect of our synthetic sample of 2 on the growth of three colorectal cancer cell lines. Growth data (relative to untreated controls but containing equimolar concentrations of DMSO carrier) are shown for HCT116 (solid line), HT29 (dashed line) and LoVo cells (dotted line), assessed by SRB assay four days after treatment. The number of cells plated per well at the beginning of the experiment is shown in parentheses. IC 50 values of 60-70 $\mu \mathrm{M}$ (HCT116), $60 \mu \mathrm{M}$ (HT29) and $50 \mu \mathrm{M}$ (LoVo) were calculated using Graphpad Prism software and are equivalent to the $\mathrm{IC}_{50}$ of $60 \mu \mathrm{M}$ reported for compound 1 by Ireland et al. ${ }^{1}$

${ }^{1} \mathrm{H}$ NMR of authentic 2-TFA alone displayed enhancement of the signals corresponding to the product whilst an impurity from authentic 2.TFA remained unchanged (Fig. S8C for selected aryl and Fig. S8D for alkyl signals, ESI $\dagger$ ). ${ }^{13}$

When reanalysed in $\mathrm{CDCl}_{3}$, our sample of 2.TFA gave chemical shifts analogous to those previously reported (Table S2, ESI $\dagger$ ). ${ }^{13}$ Comparison of the ${ }^{1} \mathrm{H}$ NMR analysis of our synthetic free base 2 in $\mathrm{CDCl}_{3}$ was also consistent with the previous literature (Table S3, ESI $\dagger$ ). ${ }^{13}$

Preliminary biological activity associated with $\mathbf{1}$ has been reported by Ireland et al., ${ }^{1}$ however, no information on the activity of $\mathbf{2}$ is available. Therefore our sample of $\mathbf{2}$ was tested against the same cancer cell line (HCT116) used to assess the activity of $\mathbf{1}$ (Fig. 2). Estimation of an $\mathrm{IC}_{50}$ inhibitory concentration showed that 2 killed HCT116 cells with similar potency to 1, suggesting that the halogens present in $\mathbf{1}$ are not essential for biological activity. Analogous studies using the colorectal cancer lines HT29 and LoVo showed that both were more sensitive to 2 than the HCT116 cells. Attempts to test the synthetic precursor N-benzyldehaloperophoramidine (30) proved difficult due to its insolubility at the concentrations used in these assays.

In conclusion, a synthesis of dehaloperophoramidine (2) from commercially available starting materials has been achieved. A highly efficient [3,3]-Claisen rearrangement to give novel ketone 6 was followed by a diastereoselective epoxide opening/ allylsilylation (modified Hosomi-Sakurai) reaction. In combination these reaction enabled construction of the required contiguous all carbon quaternary stereocentres. Detailed spectroscopic comparison with an authentic sample of 2 confirmed that it was identical to our synthetic TFA salt of 2. Preliminary biological activity associated with 2 has also been reported for the first time.

We would like to acknowledge EPSRC for PhD funding through the Doctoral Training Schemes, the EPSRC National Mass Spectrometry Service Centre, Swansea, Professor Andrew Smith for support with chiral HPLC analysis and Dr Jon Hollick for useful discussions.

\section{Notes and references}

1 S. M. Verbitski, C. L. Mayne, R. A. Davis, G. P. Concepcion and C. M. Ireland, J. Org. Chem., 2002, 67, 7124.

2 For selected reviews, see (a) B. M. Trost and M. Osipov, Chemistry, 2015, 21, 16318; (b) P. Siengalewicz, T. Gaich and J. Mulzer, Angew. Chem., Int. Ed., 2008, 47, 8170. For total syntheses of Communesins A, B and F see ; $(c)$ J. Yang, H. Wu, L. Shen and Y. Qin, J. Am. Chem. Soc., 2007, 129, 13794; (d) J. Yang, H. Wu, L. Shen and Y. Qin, Synfacts, 2008, 226; (e) P. Liu, J. H. Seo and S. M. Weinreb, Angew. Chem., Int. Ed., 2010, 49, 2000; $(f)$ Z. Zuo, W. Xie and D. Ma, J. Am. Chem. Soc., 2010, 132, 13226; $(g)$ Z. Zuo and D. Ma, Angew. Chem., Int. Ed., 2011, 50, 12008; (h) J. Belmar and R. L. Funk, J. Am. Chem. Soc., 2012, 134, 16941.

3 E. Peterson and L. E. Overman, Proc. Natl. Acad. Sci. U. S. A., 2004, $101,11943$.

4 For total and formal syntheses of perophoramidine see (a) J. R. Fuchs and R. L. Funk, J. Am. Chem. Soc., 2004, 126, 5068; (b) H. Wu, F. Xue, X. Xiao and Y. Qin, J. Am. Chem. Soc., 2010, 132, 14052; (c) H. Zhang, L. Hong, H. Kang and R. Wang, J. Am. Chem. Soc., 2013, 135, 14098; (d) S.-J. Han, F. Vogt, J. A. May, S. Krishnan, M. Gatti, S. C. Virgil and B. M. Stoltz, J. Org. Chem., 2014, 80, 528; (e) S.-J. Han, F. Vogt, S. Krishnan, J. A. May, M. Gatti, S. C. Virgil and B. M. Stoltz, Org. Lett., 2014, 16, 3316; $(f)$ B. M. Trost, M. Osipov, S. Krüger and Y. Zhang, Chem. Sci., 2015, 6, 349.

5 A. Sabahi, A. Novikov and J. D. Rainier, Angew. Chem., Int. Ed., 2006, 45, 4317.

6 T. Ishida, H. Ikota, K. Kurahashi, C. Tsukano and Y. Takemoto, Angew. Chem., Int. Ed., 2013, 52, 10204.

7 K. Popov, A. Hoang and P. Somfai, Angew. Chem., Int. Ed., 2016, $\mathbf{5 5}, 1801$.

8 N. Voûte, D. Philp, A. M. Z. Slawin and N. J. Westwood, Org. Biomol. Chem., 2010, 8, 442.

9 C. V. Ramana, R. Murali, K. Ravikumar and M. Nagarajan, J. Chem. Res (S), 1996, 5, 226.

10 For a recent example of a regioselective iodoetherification in a total synthesis, see Y. Li, S. Zhu, J. Li and A. Li, J. Am. Chem. Soc., 2016, 138, 3982 .

11 A. Hosomi and H. Sakurai, Tetrahedron Lett., 1976, 17, 1295.

12 S. V. Pansare and K. G. Kulkarni, RSC Adv., 2013, 3, 19127.

13 See ESI†.

14 C. Dallanoce, L. Rizzi, L. Pucci, C. Gotti, F. Clementi and C. De Micheli, Chirality, 2012, 24, 543.

15 CCDC 1486344 (15), CCDC 1478153 (S16), CCDC 1478152 (5) and CCDC 1478154 (30).

16 K. M. Sadhu and D. S. Matteson, Tetrahedron Lett., 1986, 27, 795.

17 (a) J. A. Ellman, T. D. Owens and T. P. Tang, Acc. Chem. Res., 2002, 35, 984; (b) M. T. Robak, M. A. Herbage and J. A. Ellman, Chem. Rev., $2010,3600$.

18 D. B. Dess and J. C. Martin, J. Org. Chem., 1983, 48, 4155.

19 (a) M. A. Mohamed, K. Yamada and K. Tomioka, Tetrahedron Lett., 2009, 50, 3436; (b) K. Yamada, Y. Mogi, M. A. Mohamed, K. Takasu and K. Tomioka, Org. Lett., 2012, 14, 5868.

20 H. Meerwein, G. Hinz, P. Hofmann, E. Kroning and E. Pfeil, J. Prakt. Chem., 1937, 147, 257.

21 S. C. Bergmeier and P. P. Seth, Tetrahedron Lett., 1999, 40, 6181. 